
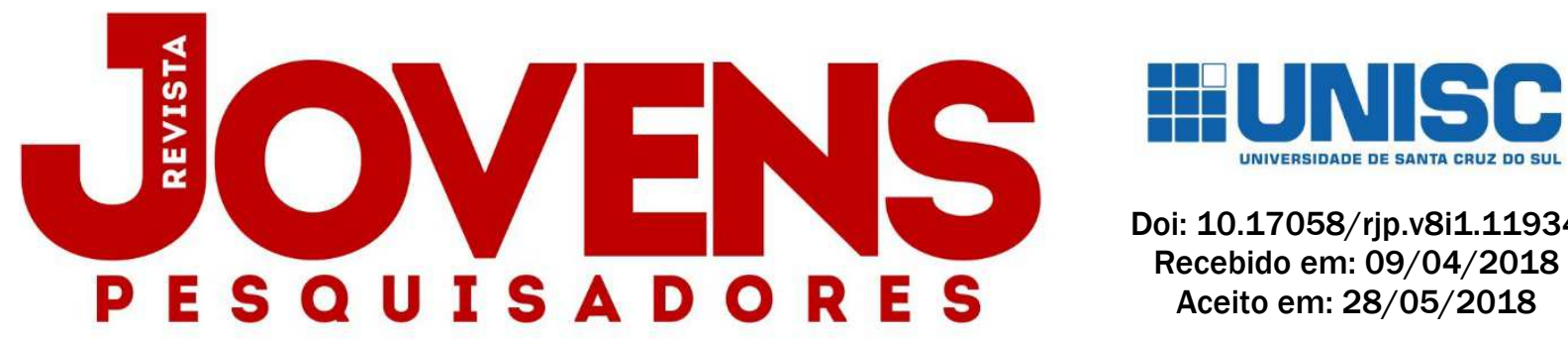

Doi: 10.17058/rjp.v8i1.11934

Recebido em: 09/04/2018

Aceito em: 28/05/2018

\title{
ASSOCIAÇÃO ENTRE ANTROPOMETRIA E PERFIL CLÍNICO COM POLIMORFISMOS NOS GENES FNDC5 RS16835198 E RBP4 RS3758539
}

\author{
RODRIGUES, S. T.1; KLINGER, E. I. ${ }^{\text {; }}$ MATOS, W. L. ${ }^{3}$; REUTER, C. P.4; TODENDI, P. F.5; VALIM, A. R. M.6
}

PALAVRAS ChAVE: Obesidade. Genes. Crianças. Adolescentes. Polimorfismo genético.

\section{RESUMO}

A prevalência de obesidade é cada vez maior em todo o mundo, principalmente em crianças e adolescentes. Essa condição tem vários fatores causais, entre eles os ambientais, nutricionais e genéticos. Para tanto, esse estudo teve como objetivo avaliar a associação entre antropometria e perfil clínico com polimorfismos (SNPs) dos genes FNDC5 rs16835198 e RBP4 rs3758539. Estudo transversal composto por 496 crianças e adolescentes, com idades entre seis e 17 anos, de escolas da rede pública (estadual e municipal) e privada do município de Santa Cruz do Sul/RS. Foram coletados dados antropométricos e realizados testes bioquímicos e clínicos. A caracterização dos genótipos foi realizada através de PCR em tempo real utilizando o sistema Taqman ${ }^{\mathrm{TM}}$. Com este estudo foi observada prevalência de excesso de peso de $36,5 \%$ dos escolares avaliados. Além disso, foi observada associação do genótipo TT do SNP rs3758539 RBP4 com maiores níveis de c-LDL, e maiores níveis de circunferência da cintura em portadores do genótipo CT.

\section{ASSOCIATION BETWEEN ANTHROPOMETRY AND CLINICAL PROFILE WITH POLYMORPHISMS IN FNDC5 GENES RS16835198 AND RBP4 RS3758539}

KEYWORDS: Obesity. Genes. Children. Adolescents. Genetic polymorphism.

\begin{abstract}
The prevalence of obesity is increasing worldwide, especially in children and adolescents. This condition has several causal factors, including environmental, nutritional and genetic factors. Therefore, this study aimed to evaluate the association between anthropometry and clinical profile with polymorphisms (SNPs) of FNDC5 genes rs16835198 and RBP4 rs3758539. A cross-sectional study composed of 496 children and adolescents, aged between six and 17 years, from public (state and municipal) and private schools in the city of Santa Cruz do Sul/RS. Anthropometric data were collected and biochemical and clinical tests were measured. The genotypes characterization were done by real time PCR using the Taqman system ${ }^{\text {TM }}$. With this study, prevalence of overweight was observed in $36.5 \%$ of the students evaluated. In addition, we observed an association of the $\Pi$ genotype of rs3758539 RBP4 SNP with higher levels of LDL-C, and higher levels of waist circumference in patients with the CT genotype.
\end{abstract}

1 Graduada em Farmácia pela Universidade de Santa Cruz do Sul.

2 Graduada em Farmácia e Mestranda em Promoção da Saúde na Universidade de Santa Cruz do Sul.

${ }^{3}$ Acadêmico do curso de Farmácia na Universidade de Santa Cruz do Sul.

4 Docente do Departamento de Educação Física e Saúde da Universidade de Santa Cruz do Sul.

${ }^{5}$ Graduada em Farmácia e Mestre em Promoção da Saúde pela Universidade de Santa Cruz do Sul.

${ }^{6}$ Docente do Departamento de Biologia e Farmácia na Universidade de Santa Cruz do Sul. 


\section{INTRODUÇÃO}

De acordo com a análise sistemática de prevalência global, regional e nacional de sobrepeso e obesidade, em crianças e adultos durante o período de 1980 a 2013, realizada por Marie et al. (2014), a prevalência combinada aumentou substancialmente $27,5 \%$ para adultos e $47,1 \%$ para crianças. Em outra pesquisa, verificou-se que $40 \%$ da população americana enquadra-se aos critérios para obesidade (YEH et al., 2016). Embora a prevalência entre crianças e adolescentes seja menor que em adultos, a taxa de aumento da obesidade infantil vem sendo maior que a taxa de aumento da obesidade em adultos (ROTH; JAIN, 2018). Isso também é mostrado no estudo de Derén et al. (2018) realizado na Ucrânia, em que foi observada maior prevalência de sobrepeso e obesidade na população mais jovem, comparando a estudos com sujeitos mais velhos.

Reconhecida como uma pandemia mundial, a obesidade infanto-juvenil requer além de estratégias para o controle, estudos que abordem sua ampla complexidade, mostrando a relação do efeito genético, condições ambientais, e possíveis consequências como as alterações metabólicas (DABAS; SETH, 2018; JANG et al., 2017). 0 IMC elevado representa uma das maiores causas de morte, principalmente em decorrência de doenças cardiovasculares (AFSHIN et al., 2017).

Os fatores genéticos são cada vez mais estudados quando se fala em obesidade (ORNELLAS et al., 2017). Ao examinarem o impacto de polimorfismos de nucleotídeos únicos (SNP) em genes associados à obesidade e suas interações em crianças com idade escolar na China, Zhao et al. (2013) puderam notar que o risco genético foi associado ao risco de obesidade $(p<0,001)$. As diferenças nos níveis de lipídios séricos entre indivíduos com peso normal e sobrepeso ou obesos também podem ser consequências, em parte, de diferentes polimorfismos genéticos e das interações entre vários SNPs (YIN et al., 2012).

A obesidade é considerada uma doença poligênica, em que a suscetibilidade de cada indivíduo é determinada por interações entre o código genético com fatores ambientais e comportamentais, conferindo diferentes manifestações (PIANTINO et al., 2017). Para tanto, esse estudo teve como objetivo avaliar a associação entre antropometria e perfil clínico com polimorfismos nos genes FNDC5 rs16835198 e RBP4 rs3758539.

\section{FUNDAMENTAÇÃO TEÓRICA}

Além dos fatores ambientais, existem variantes genéticas que afetam o risco de obesidade, sendo determinadas em estudos de associação genômica de Polimorfismo de Nucleotídeo Único (SNPs) comuns como o RBP4 e a Irisina (FNDC5) (SCOTT et al., 2016). Para a escolha de medidas específicas e efetivas para a prevenção da obesidade há a necessidade de se conhecer a interação entre os componentes ambientais e genéticos (PEREIRA et al., 2016). 
Dentre vários fatores genéticos que estão relacionados com a obesidade, a Irisina vem sendo muito estudada. Trata-se de um produto da clivagem proteolítica de FNDC5 cuja expressão é induzida pelo co-ativador PGC1-a, e esta expressão é induzida pelo exercício físico que estimula a liberação da Irisina para a circulação (MORENO et al., 2015). A Irisina é secretada durante a contração muscular esquelética e media alguns benefícios para a saúde por meio do "escurecimento" do tecido adiposo branco (KURDIOVA et al., 2014). Embora se saiba que a principal fonte de Irisina é no músculo, já se detectou sua presença no tecido adiposo subcutâneo e visceral (NOVELLE et al., 2013). A Irisina pode desempenhar um papel muito importante na redução do risco de obesidade, e ainda, seus altos níveis contribuem para um envelhecimento saudável dos seres humanos. Recentemente foi demonstrado que SNPs no gene FNDC5 rs16835198 e rs726344, estão associados à sensibilidade à insulina em adultos, trazendo riscos à saúde (SANCHIS-GOMAR, 2014).

Outro gene, possível contribuinte do agravo da obesidade e suas comorbidades é a proteína 4 de ligação ao retinol (RBP4), sendo relatada sua associação à adiposidade, resistência à insulina e o diabetes mellitus tipo 2 (DM2). 0 papel fisiológico da RBP4 é o transporte do retinol do fígado para os tecidos periféricos. Um dos primeiros eventos relacionados ao acúmulo excessivo de gordura visceral é o desenvolvimento de inflamação seguida de disfunção hormonal do tecido adiposo, incluindo a produção demasiada de RBP4 (LU et al., 2016).

A RBP4 circulante inibe as vias de sinalização estimuladas pela insulina nas células do músculo esquelético, resultando no desenvolvimento de resistência à insulina. 0 aumento da secreção de RBP4 também estimula a expressão de moléculas de adesão nas células endoteliais, promovendo o desenvolvimento de aterosclerose e hipertensão arterial. Estudos populacionais demonstraram uma associação entre a RBP4 sérica e os SNPs funcionais na gravidade da aterosclerose elevando o risco de eventos cardiovasculares e DM2 (MAJERCZYK et al., 2016).

\section{MATERIAIS E MÉTODOS}

Trata-se de um estudo transversal realizado no município de Santa Cruz do Sul/RS nos anos de 2014 e 2015. Os sujeitos do estudo compõem aproximadamente 496 crianças e adolescentes, com idades entre seis e 17 anos, de escolas da rede pública (estadual e municipal) e privada. Este estudo atende os referenciais básicos da Bioética, previstos na Resolução 466/2012 do Conselho Nacional de Saúde, sobre pesquisas envolvendo seres humanos. Dentre as variáveis descritivas aplicadas a estes escolares inclui-se sexo, faixa etária e etnia/cor que foram autorreferidas em questionário.

Foram avaliadas as medidas antropométricas como Índice de Massa Corporal (IMC) classificado a partir das curvas de percentis da Organização Mundial da Saúde (WHO, 2007), de acordo com sexo e idade, considerando baixo peso ( $<p 3)$, normal ( $\geq p 3$ e $<p 85)$, sobrepeso ( $\geq p 85$ e 
<p97) e obesidade ( $\geq$ p97). A Circunferência da Cintura (CC), avaliada através de fita métrica inelástica, utilizando como referência a parte mais estreita do tronco entre as costelas e a crista ilíaca e o quadril no nível do trocanter maior, sendo posteriormente classificada através dos critérios estabelecidos por Fernández et al. (2004), de acordo com sexo e idade, considerando circunferência normal percentil $<75$ e elevado com percentil $\geq 90$. E Percentual de Gordura (\%G) calculado através da equação de Slaugther et al. (1988), sendo posteriormente classificado de acordo com os dados de Lohman (1987) e separado em duas categorias: muito baixo/baixo/ótimo e moderadamente alto/alto/muito alto.

As amostras sanguíneas foram coletadas após 12 horas de jejum. Os parâmetros bioquímicos e clínicos analisados foram Colesterol Total (CT), Colesterol LDL (c-LDL), Colesterol HDL (c-HDL), Triacilgliceróis (TAG) e Glicose. 0 c-LDL foi calculado usando a equação de Friedewald, Levy e Fredrickson (1972) e os demais dosados em equipamento automatizado Miura One (ISE, Roma, Itália). De acordo com o Instituto Nacional do Coração, Pulmão e Sangue (2011), consideramos como ponto de corte para cada parâmetro os seguintes valores: CT: $\geq 200 \mathrm{mg} / \mathrm{dL}, \mathrm{C}-\mathrm{LDL}: \geq 130 \mathrm{mg} / \mathrm{dL}, \mathrm{c}-$ HDL: <40 mg / dL, TAG: $\geq 100$ mg / dL (0-9 anos) ou $\geq 130$ mg / dL (10-19 anos) e glicose: < 99 mg / dL (normal), 100-125 mg / dL (tolerância a glicose diminuída) e > $126 \mathrm{mg}$ / dL (Diabetes). Também foram aferidas a Pressão Arterial Sistólica (PAS) e Pressão Arterial Diastólica (PAD).

Para a análise de genotipagem, primeiramente foi extraído DNA a partir de $500 \mu \mathrm{L}$ das amostras de sangue total anticoagulado com EDTA através do método de Salting out, conforme descrito por Miller, Dykes e Polesky (1988). Depois da extração, o DNA foi ressuspendido em água ultra-pura, quantificado no aparelho NanoDrop 2000c Spectrophotometer (Thermo Scientific, Wilmington, EUA) e armazenado à $-20^{\circ} \mathrm{C}$. O DNA extraído foi utilizado em uma reação de qPCR (Reação em Cadeia da Polimerase em tempo real), no StepOne Plus ${ }^{\circledR}$ (Applied Biosystems, Foster City, CA, EUA), conforme instruções do fabricante, utilizando ensaios de discriminação alélica. Para o gene FNDC5 foi analisado o SNP rs16835198 através do ensaio C_34204885_10 e para o SNP rs3758539 do gene RBP4 o ensaio utilizado foi C__11756910_10.

Os dados foram analisados no programa estatístico SPSS v. 20. A descrição da amostra foi expressa através de uma análise descritiva para verificar os escolares com IMC normal, sobrepeso e obesidade em cada um dos grupos das variáveis relacionadas com o perfil lipídico, glicêmico e polimorfismos genéticos. Foi utilizado o teste Kolmogorov-Smirnov (K-S) para diferenciar as amostras paramétricas e não paramétricas. 0 equilíbrio de Hardy-Weinberg (HWE) foi analisado para todos os polimorfismos. A associação entre as variáveis foi realizada através de comparação de médias das variáveis antropométricas e bioquímicas com os SNPs, através de ANOVA. 


\section{RESULTADOS E DISCUSSÃO}

Obesidade na infância e na adolescência está associada com resistência à insulina, metabolismo anormal da glicose, hipertensão arterial, dislipidemia, inflamação, doença hepática e função vascular comprometida. Por isso, é importante avaliar essa faixa etária buscando alternativas para a prevenção e tratamento das comorbidades relacionadas a essa condição (MANSOUR et al., 2016). A população avaliada no presente estudo foi constituída por $56,3 \%$ dos sujeitos do sexo feminino e 79,4\% eram adolescentes com idade mediana de 12 anos (IIQ: 10-14).

Destaca-se ainda que $69,0 \%$ dos escolares se autodeclararam de etnia/cor branca e que $36,5 \%$ apresentaram de excesso de peso (Tabela 1). Esse dado evidencia um incremento quando comparado ao estudo anterior com a mesma população do município de Santa Cruz do Sul, cuja prevalência foi de $27 \%$ de excesso de peso em 729 escolares analisados (BARBIAN et al., 2017). Um estudo realizado em Porto Alegre (RS) com crianças do $1^{\circ}$ ao $4^{\circ}$ ano do ensino fundamental revelou que 38,4\% dos avaliados apresentavam excesso de peso (FRIEDRICH, ANTUNES, SCHUCH, 2016).

Globalmente o excesso de peso entre crianças e adolescentes também vem chamando a atenção. Na China, com uma amostra de 49.549 crianças em idade escolar, um estudo de prevalência mostrou que $\mathbf{2 4 , 9 \%}$ dos participantes estavam acima do peso (ZHU et al., 2018). STIEFEL et al. (2016), no estado de Mississippi (EUA), observaram uma prevalência de $23,5 \%$ de obesidade e 20,3\% de sobrepeso ao avaliarem 7.705 adolescentes de 14 a 18 anos. 
Tabela 1. Características antropométricas e clínicas dos escolares.

\begin{tabular}{|c|c|c|c|c|}
\hline & $n=496(\%)$ & $\begin{array}{c}\begin{array}{c}\text { Não- } \\
\text { obesos }\end{array} \\
n=315 \\
(63,5 \%)\end{array}$ & $\begin{array}{c}\text { Obesos/Sobrepes } \\
\text { o } \\
n=182(36,5 \%)\end{array}$ & $p$ \\
\hline Sexo & & & & 0,456 \\
\hline Feminino & $279(56,3)$ & $\begin{array}{c}185 \\
(58,7)\end{array}$ & $94(51,9)$ & \\
\hline Masculino & $217(43,8)$ & $\begin{array}{c}130 \\
(41,3)\end{array}$ & $87(48,1)$ & \\
\hline Faixa Etária† & $12,00(10-14) *$ & & & 0,132 \\
\hline Criança & $102(20,6)$ & $56(17,8)$ & $46(25,4)$ & \\
\hline Adolescente & $394(79,4)$ & 259 & $135(74,6)$ & \\
\hline Etnia/Cor & & $(82,2)$ & & 0,737 \\
\hline Branco & $342(69,0)$ & & $130(71,8)$ & \\
\hline Negro & $45(9,1)$ & 212 & $17(9,4)$ & \\
\hline Parda/Mulata & $96(19,4)$ & $(67,3)$ & $30(16,6)$ & \\
\hline Indígena & $5(1,0)$ & $28(8,9)$ & $2(1,1)$ & \\
\hline \multirow[t]{3}{*}{ Amarelo } & $8(1,6)$ & $66(21,0)$ & $2(1,1)$ & \\
\hline & & $3(1,0)$ & & \\
\hline & & $6(1,9)$ & & \\
\hline $\mathrm{CC}(\mathrm{cm})$ & $65,35(60,0-73,82)^{*}$ & & & $<0,001$ \\
\hline Normal & $377(76,0)$ & $\begin{array}{c}298 \\
(94,6)\end{array}$ & $79(43,6)$ & \\
\hline Elevada & $119(24,0)$ & $17(5,4)$ & $102(56,4)$ & \\
\hline$\% G$ & $20,7(15,6-26,7)^{*}$ & & & $<0,001$ \\
\hline Muito baixo, baixo e ótimo & $306(61,7)$ & $\begin{array}{c}256 \\
(81,3)\end{array}$ & $50(27,6)$ & \\
\hline $\begin{array}{c}\text { Moderadamente alto, alto e } \\
\text { muito alto }\end{array}$ & $190(38,4)$ & $59(18,7)$ & $131(72,4)$ & \\
\hline Colesterol Total (mg/dL) & $163,07( \pm 31,43) * *$ & & & 0,274 \\
\hline Normal & $302(60,9)$ & $\begin{array}{c}201 \\
(63,8)\end{array}$ & $101(55,8)$ & \\
\hline Limítrofe & $138(27,8)$ & $80(25,4)$ & $58(32,0)$ & \\
\hline Aumentado & $56(11,3)$ & $34(10,8)$ & $22(12,2)$ & \\
\hline Colesterol LDL (mg/dL) & $84,93( \pm 28,43) * *$ & & & 0,200 \\
\hline Normal & $404(81,5)$ & $\begin{array}{c}267 \\
(84,8)\end{array}$ & $137(75,7)$ & \\
\hline Limítrofe & $65(13,1)$ & $34(10,8)$ & $31(17,1)$ & \\
\hline Aumentado & $27(5,4)$ & $14(4,4)$ & $13(7,2)$ & \\
\hline Colesterol HDL (mg/dL) & $62,78( \pm 11,16)$ ** & & & 0,001 \\
\hline Normal & $464(93,5)$ & $\begin{array}{c}305 \\
(96,8)\end{array}$ & $159(87,8)$ & \\
\hline Limítrofe & $26(5,2)$ & $8(2,5)$ & $18(9,9)$ & \\
\hline Baixo & $6(1,2)$ & $2(0,6)$ & $4(2,2)$ & \\
\hline Triacilgliceróis (mg/dL) & $68,2(51,3-91,8) *$ & & & $<0,001$ \\
\hline Normal & $351(70,8)$ & $\begin{array}{c}238 \\
(75,6)\end{array}$ & $113(62,4)$ & \\
\hline Limítrofe & $102(20,6)$ & $59(18,7)$ & $43(23,8)$ & \\
\hline Aumentado & $43(8,7)$ & $18(5,7)$ & $25(13,8)$ & \\
\hline Glicose (mg/dL) & $90,0(86,0-95,0) *$ & & & 0,121 \\
\hline Normal & $433(87,3)$ & $\begin{array}{c}269 \\
(85,4)\end{array}$ & $164(90,6)$ & \\
\hline Limítrofe & $62(12,5)$ & $46(14,6)$ & $16(8,8)$ & \\
\hline Elevado & $1(0,2)$ & $0(0,0)$ & $1(0,6)$ & \\
\hline PAS & $105,0(100,0-120,0)$ * & & & $<0,001$ \\
\hline Normal & $395(79,6)$ & 272 & $123(68,0)$ & \\
\hline Elevado & $101(20,4)$ & $(86,3)$ & $58(32,0)$ & \\
\hline PAD & $63,5(60,0-70,0)^{*}$ & $43(13,7)$ & & 0,001 \\
\hline Normal & $420(84,7)$ & & $139(76,8)$ & \\
\hline Elevado & $76(15,3)$ & $\begin{array}{c}281 \\
(89,2) \\
34(10,8)\end{array}$ & $42(23,2)$ & \\
\hline
\end{tabular}

†Faixa Etária: criança <10 anos, adolescente 10-17 anos. * Valores expressos em mediana e intervalo interquartil (IIQ). * * Valores expressos em média (Desvio-padrão). 
Os escolares apresentaram CC mediana de 65,35 cm (IIQ: 60,0-73,82) e \%G de 20,7 (IIQ: 15,6-26,7). Essa variável em sujeitos obesos ou com sobrepeso revelou que $56,4 \%$ dos escolares apresentavam alteração, e 72,4\% dos indivíduos que apresentaram obesidade e sobrepeso tiveram \%G moderadamente alto, alto e muito alto. A comparação da CC e \%G entre os sujeitos classificados como obesos ou com sobrepeso apresentou diferença estatisticamente significativa em relação aos não obesos ( $p<0,001)$. Um estudo epidemiológico transversal com 930 indivíduos, que incluiu adolescentes de 14 a 19 anos, desenvolvido na cidade de São José, SC, verificou uma prevalência de obesidade abdominal de 10,6\% (CASTRO; NUNES; SILVA, 2016), bem inferior ao observado no presente estudo que foi 24\%. Em um estudo transversal realizado na Espanha com 149 indivíduos, sendo estes classificados como saudáveis e obesos, de acordo com o IMC, verificou-se também a elevação significativa do \%G e glicose no grupo com sobrepeso e obesidade em relação ao grupo saudável ( $p>0,001$ ) (CHIELLE et al., 2017). No presente estudo não foi observada diferença significativa dos níveis médios de glicose entre os sujeitos com excesso de peso e os sem obesidade.

A média de c-HDL do conjunto escolares avaliados foi de $62,78 \mathrm{mg} / \mathrm{dL}( \pm 11,16)$ e TAG de $68,2 \mathrm{mg} / \mathrm{dL}$ (IIQ: 51,3-91,8), sendo que a comparação entre os sujeitos obesos ou com sobrepeso, com não obesos resultou em diferença estatística significativa ( $p \leq 0,001)$. Entre escolares não obesos, os níveis de c-HDL se mostraram normais em $96,8 \%$ da amostra estudada. Os níveis de TAG aumentados entre os escolares obesos e/ou sobrepeso foram prevalentes em 13,8\%. Em um estudo transversal que envolveu 840 crianças e adolescentes classificados em não obesos e obesos de acordo com o IMC, foram também observadas diferenças estatísticas significativas nas médias de cLDL ( $p=0,003)$, c-HDL, TAG e \%g (<0,001) (OLIOSA et al., 2017).

Relacionado à pressão arterial, foi observada para o conjunto de sujeitos analisados a mediana da PAS de 105,0 mmHg (IIQ: 100,0-120,0) e a PAD de 63,5 mmHg (IIQ: 60,0-70,0). Além disso, a comparação entre os valores médios dos sujeitos não obesos com os obesos e com sobrepeso revelou diferença entre os grupos ( $p \leq 0,001)$, evidenciando maior risco cardiovascular em sujeitos com sobrepeso e obesidade desde muito jovens. A PAS elevada dessas crianças e adolescentes foi prevalente em $20,4 \%$ do total da amostra abordada no estudo, já entre obesos/sobrepeso 32,0\% apresentaram PAS elevada ( $p<0,001)$. E quanto a PAD, 15,3\% da amostra total apresentou elevação, e entre obesos/sobrepeso, 23,2\% destes indivíduos apresentaram PAD elevada. Mudie et al. (2018) concluíram em seu estudo de base populacional realizado em Malawi, país da África Oriental, que o IMC está fortemente associado ao risco cardiometabólico $(p<0,001)$ principalmente em residentes de áreas urbanas, destacando a importância de avaliar o risco nessas populações.

A prevalência de pressão arterial elevada nessa faixa etária também foi observada em um estudo realizado com crianças e adolescentes de ambos os sexos dos Emirados Árabes Unidos (EAU), em que a PAS elevada se deu em 14,6\% da amostra e a PAD elevada em 4,9\% (ABDULLE et al., 
2014). E entre crianças com sobrepeso e obesidade avaliadas em um estudo em Shanghai, China, a prevalência de PAS elevada foi de 13,29\% (sobrepeso) e 21,63\% (obesidade), da mesma forma, a PAD elevada foi identificada em 7,70\% (sobrepeso) e 13,41\% (obesidade), tendo $p<0,001$ para todos estes resultados (LU et al., 2013).

A distribuição das frequências genotípicas e alélicas observadas para os SNPs analisados estavam em equilíbrio de Hardy-Weinberg $(p>0,05)$ (Tabela 2). 0 genótipo que apresentou maior frequência no gene FNDC5 foi o GT (45,8\%) seguido do genótipo GG (41,5\%) e o alelo mais frequente foi o alelo G (64,42\%). Resultados similares são apresentados no estudo de Khidr et al. (2017) realizado no Egito, que verificou uma prevalência genotípica de 42\% para GT, 36\% para GG e $22 \%$ para $\Pi$, e ainda uma frequência maior do alelo $G(57 \%)$. Quanto ao RBP4, observou-se frequência de $3,6 \%$ do genótipo de risco $\Pi \mathrm{e}$ e 16,73\% do alelo T (Tabela 2). Resultados semelhantes a um estudo que verificou a frequência genotípica e alélica em mulheres caucasianas, em que a frequência do genótipo $T$ foi de $3,2 \%(p=0,115)$ e a frequência do alelo $T$ foi de $15,3 \%(p=0,489)$ (HIRAOKA et al., 2011).

Tabela 2. Frequência genotípica e alélica dos SNPs.

\begin{tabular}{|c|c|c|c|}
\hline & $\begin{array}{c}\text { Frequência observada } \\
n=496(\%)\end{array}$ & $\begin{array}{c}\text { Frequência esperada } \\
n=496(\%)\end{array}$ & $p$ \\
\hline FNDC5 rs16835198 & & & 1,000 \\
\hline GG & $206(41,5)$ & $206(41,5)$ & \\
\hline GT & $227(45,8)$ & $227(45,8)$ & \\
\hline$\pi$ & $63(12,7)$ & $63(12,7)$ & \\
\hline G & $639(64,42)$ & & \\
\hline $\mathrm{T}$ & $353(35,58)$ & & \\
\hline RBP4 rs3758539 & & & 0,438 \\
\hline CC & $348(70,2)$ & $344(69,3)$ & \\
\hline CT & $130(26,2)$ & $138(27,8)$ & \\
\hline$\pi$ & $18(3,6)$ & $14(2,8)$ & \\
\hline C & $826(83,26)$ & & \\
\hline$T$ & $166(16,73$ & & \\
\hline
\end{tabular}

Com relação às medidas antropométricas, perfil bioquímico e clínico dos escolares (Tabela 3), podemos observar que os portadores do genótipo GG do SNP FNDC5 rs16835198 apresentaram maior mediana de PAD, 70,00 $\mathrm{mmHg}(60,00-70,00)$ em relação aos outros genótipos, $\Pi$ e GT ( $p=0,050)$. E quanto ao SNP RBP4 rs3758539, portadores do genótipo CT apresentaram maior CC $(p=0,046)$ e os portadores do genótipo $T$ apresentaram maior média de c-LDL $(100,36 \pm 32,36)$ em relação aos outros genótipos $\mathrm{CT}$ e CC, sendo $p=0,044$. Na mesma linha do presente estudo, porém com diferentes variáveis, Codoñer-Franch et al. (2015) examinaram a associação potencial de quatro SNPs do gene RBP4 com a obesidade infantil e suas complicações metabólicas, em que observou-se a associação do alelo C do SNP RBP4 rs3758539 (A>C) com a obesidade e resistência a insulina. 
Tabela 3. Relação dos dados antropométricos, bioquímicos e clínicos com os SNPs analisados.

\begin{tabular}{|c|c|c|c|c|c|c|c|c|}
\hline & \multicolumn{3}{|c|}{ FNDC5_rs16835198 } & \multirow{2}{*}{$p$} & \multicolumn{3}{|c|}{ RBP4_rs3758539 } & \multirow{2}{*}{$p$} \\
\hline & $\pi$ & GT & GG & & $\pi$ & CT & CC & \\
\hline IMC* & $\begin{array}{c}21,3(17,5- \\
23,3)\end{array}$ & $\begin{array}{c}19,5(17,5- \\
22,2)\end{array}$ & $\begin{array}{c}20,3(17,2- \\
23,6)\end{array}$ & 0,300 & $\begin{array}{c}18,5(15,6- \\
25,5)\end{array}$ & $\begin{array}{c}21,0(18,6- \\
24,2)\end{array}$ & $\begin{array}{c}19,5(17,2- \\
22,9)\end{array}$ & 0,065 \\
\hline $\mathrm{CC}(\mathrm{cm}) *$ & $\begin{array}{c}66,0(59,0- \\
76,0)\end{array}$ & $\begin{array}{c}65,0(60,0- \\
72,0)\end{array}$ & $\begin{array}{c}66,0(60,0- \\
75,0)\end{array}$ & 0,340 & $\begin{array}{c}64,0(57,5- \\
74,6)\end{array}$ & $\begin{array}{c}67,0(60,2- \\
77,0)\end{array}$ & $\begin{array}{c}65,0(59,0- \\
72,0)\end{array}$ & 0,046 \\
\hline$\% G(\%)^{*}$ & $\begin{array}{c}22,4(15,7- \\
27,6)\end{array}$ & $\begin{array}{c}20,0(15,6- \\
26,4)\end{array}$ & $\begin{array}{c}21,1(15,4- \\
27,2)\end{array}$ & 0,474 & $\begin{array}{c}17,6(11,8- \\
29,0)\end{array}$ & $\begin{array}{c}21,2(16,5- \\
26,8)\end{array}$ & $\begin{array}{c}19,9(15,4- \\
26,7)\end{array}$ & 0,332 \\
\hline $\begin{array}{c}\mathrm{HDL} \\
(\mathrm{mg} / \mathrm{dL}) * *\end{array}$ & $\begin{array}{c}61,89 \\
( \pm 12,95)\end{array}$ & $\begin{array}{c}63,62 \\
( \pm 10,25)\end{array}$ & $\begin{array}{c}62,13 \\
( \pm 11,53)\end{array}$ & 0,303 & $\begin{array}{c}60,27 \\
(13,11)\end{array}$ & $\begin{array}{c}62,60 \\
(10,41)\end{array}$ & $\begin{array}{c}62,98 \\
(11,35)\end{array}$ & 0,590 \\
\hline $\begin{array}{c}\mathrm{LDL} \\
(\mathrm{mg} / \mathrm{dL})^{* *}\end{array}$ & $\begin{array}{c}81,54 \\
(28,11)\end{array}$ & $\begin{array}{c}85,78 \\
( \pm 28,85)\end{array}$ & $\begin{array}{c}85,04 \\
( \pm 28,13)\end{array}$ & 0,577 & $\begin{array}{l}100,36 \\
(32,36)\end{array}$ & $\begin{array}{c}86,20 \\
(27,77)\end{array}$ & $\begin{array}{c}83,66 \\
(28,29)\end{array}$ & 0,044 \\
\hline $\begin{array}{c}\mathrm{CT} \\
(\mathrm{mg} / \mathrm{dL}) * *\end{array}$ & $\begin{array}{l}158,79 \\
(30,98)\end{array}$ & $\begin{array}{c}165,28 \\
( \pm 31,60)\end{array}$ & $\begin{array}{c}161,95 \\
( \pm 31,34)\end{array}$ & 0,280 & $\begin{array}{l}177,38 \\
(34,78)\end{array}$ & $\begin{array}{l}164,57 \\
(30,60)\end{array}$ & $\begin{array}{l}161,77 \\
(31,44)\end{array}$ & 0,099 \\
\hline $\begin{array}{c}\text { TAG } \\
(\mathrm{mg} / \mathrm{dL}) *\end{array}$ & $\begin{array}{c}69,7(52,4- \\
90,8)\end{array}$ & $\begin{array}{c}70,2(53,0- \\
91,9)\end{array}$ & $\begin{array}{c}66,4(49,2- \\
92,3)\end{array}$ & 0,252 & $\begin{array}{c}74,9(48,8- \\
101,3)\end{array}$ & $\begin{array}{c}64,9(46,4- \\
91,7)\end{array}$ & $\begin{array}{c}69,2(52,3- \\
91,5)\end{array}$ & 0,531 \\
\hline $\begin{array}{c}\text { Glicose } \\
\text { (mg/dl)* }\end{array}$ & $\begin{array}{c}90,0(85,0- \\
94,0)\end{array}$ & $\begin{array}{c}91,0(86,0- \\
96,0)\end{array}$ & $\begin{array}{c}90,0(86,0- \\
95,0)\end{array}$ & $\begin{array}{l}0,270 \\
0,058\end{array}$ & $\begin{array}{c}92,0(86,0- \\
97,0)\end{array}$ & $\begin{array}{c}90,0(86,0- \\
95,0)\end{array}$ & $\begin{array}{c}90,0(86,0- \\
95,0)\end{array}$ & $\begin{array}{l}0,732 \\
0,502\end{array}$ \\
\hline \multirow[t]{2}{*}{ PAS* } & 105,0 & 102,0 & 110,0 & & 103,0 & 108,0 & 105,0 & \\
\hline & $\begin{array}{c}(100,0- \\
120,0)\end{array}$ & $\begin{array}{l}(95,0- \\
110,0)\end{array}$ & $\begin{array}{c}(100,0- \\
120,0)\end{array}$ & 0,050 & $\begin{array}{l}(97,5- \\
122,5)\end{array}$ & $\begin{array}{l}(100,0- \\
120,0)\end{array}$ & $\begin{array}{l}(96,0- \\
115,0)\end{array}$ & 0,852 \\
\hline PAD* & $\begin{array}{c}60,0(60,0- \\
70,0)\end{array}$ & $\begin{array}{c}60,0(60,0- \\
70,0)\end{array}$ & $\begin{array}{c}70,0(60,0- \\
70,0)\end{array}$ & & $\begin{array}{c}62,5(50,0- \\
72,5)\end{array}$ & $\begin{array}{c}60,0(60,0- \\
70,0)\end{array}$ & $\begin{array}{c}65,0(60,0- \\
70,0)\end{array}$ & \\
\hline
\end{tabular}

* Valores expressos em mediana e intervalo interquartil (IIQ). ** Valores expressos em média (Desvio-padrão)

\section{CONSIDERAÇÕES FINAIS}

Este estudo mostrou alta prevalência de sobrepeso e obesidade, bem como, as significativas alterações nas medidas de CC e \%G, níveis de c-HDL, TAG, PAS e PAD em crianças e adolescentes com excesso de peso, comparadas a sujeitos não obesos. Também foi percebida uma associação do SNP rs3758539 RBP4 com maiores valores de CC e c-LDL nos indivíduos avaliados.

\section{AGRADECIMENTOS}

Agradecemos à Universidade de Santa Cruz do Sul - UNISC, à Fundação de Amparo à Pesquisa do Estado do Rio Grande do Sul (FAPERGS) e a Professora Dra. Miria Suzana Burgos (in memoriam). 


\section{REFERÊNCIAS}

ABDULle, A., AL-JUNAIBI, A., NAGELKERKE, N. High Blood Pressure and Its Association with Body Weight among Children and Adolescents in the United Arab Emirates. Plos one, San Francisco, v. 9, n. 1, 2014.

AFSHIN, A. et al. Health Effects of Overweight and Obesity in 195 Countries over 25 Years. The new England Journal of Medicine, v. 377, p. 13-27, 2017.

BARBIAN, C. D. et al. Comparison of the nutritional, lipidic and glycemic profile of children and adolescents of different hemispheres of the rural area of Santa Cruz do Sul - RS. CINERGIS, Santa Cruz do Sul, v. 18, n. 2, 2017.

CASTRO, J. A. C. NUNES, H. E. G. SILVA, D. A. S. Prevalence of abdominal obesity in adolescents: association between sociodemographic factors and lifestyle. Revista Paulista de Pediatria. V. 34, n. 3, p. 243-251, 2016.

CHIELLE, E. 0 . et al. Influence of obesity on the serum concentration of retinol-binding protein 4 (RBP4) in young adults. Jornal Brasileiro de Patologia e Medicina Laboratorial. Rio de Janeiro, v. 53, n. 2, p. 81-86, 2017.

CODOÑER-FRANCH, P. et al. Association of RBP4 genetic variants with childhood obesity and cardiovascular risk factors. Pediatric Diabetes, v. 17, n. 8, 2015.

DABAS, A. SETH, A. Prevenção e gestão da obesidade infantil. Indian Journal of Pediatrics, v. 0, n. 0 , 2018.

DERÉN, K. et al. The prevalence of underweight, overweight and obesity in children and adolescents from Ukraine. Scientific Reports, v, 8, n. 3625, 2018.

FERNÁNDEZ, J. R. et al. Waist circumference percentiles in nationally representative samples of African-American, European-American, and Mexican-American children and adolescents. The Journal of Pediatrics, Cincinnati, v. 145, n. 4, p. 439-444, 2004.

FRIEDRICH, R. R.; ANTUNES, A.; SCHUCH, I. Percepção materna do estado nutricional de crianças em escolas municipais de Porto Alegre-RS. Demetra, Rio de Janeiro, v. 11, n. 1, p. 211-223, 2016.

FRIEDEWALD, W. T.; LEVY, R. I.; FREDRICKSON, D. S. Estimation of the concentration of low-density lipoprotein cholesterol in plasma, without use of the preparative ultracentrifuge. Clinical Chemisty, Baltimore, v. 18, n. 6, p. 499-502, 1972.

HIRAOKA, M. et al. A Polymorphism in the Retinol Binding Protein 4 Gene is Not Associated with Gestational Diabetes Mellitus in Several Different Ethnic Groups. Hawai'l Medical Journal, Hawai, v. 70, n. 8, p. 164-167, 2011.

Instituto Nacional do Coração, Pulmão e Sangue. NHLBI. Painel de especialistas em diretrizes integradas para saúde cardiovascular e redução de risco em crianças e adolescentes. Bethesda: 2012.

KHIDR, E. G. Association of irisin and FNDC5 rs16835198 G > T gene polymorphism with type 2 diabetes mellitus and diabetic nephropathy. An Egyptian pilot study. Gene, v. 626, p. 26-31, 2017.

KURDIOVA, T. et al. Effects of obesity, diabetes and exercise on Fndc5 gene expression and irisin release in human skeletal muscle and adipose tissue: in vivo and in vitro studies. The Journal of Physiology. v. 592, n. 5, p. 1091- 1107, 2014.

LOHMAN, Timothy. The use of skinfold to estimate body fatness on children and youth. Journal of Physical Education, Recreation e Dance, London, v. 58, n. 9, p. 98-102, 1987.

LU, X. et al. Prevalence of hypertension in overweight and obese children from a large school-based population in Shanghai, China. BMC Public Health, Londres, v. 13, n. 24, 2013.

LU, Z. et al. Serum levels of RBP4 might not be determined by diabetes mellitus but by kidney function and renal replacement therapy. Journal of Shanghai Jiaotong University. v. 21, n. 6, p. 757762, Dez., 2016.

MAJERCZYK, M. et al. Retinol-binding protein 4 (RBP4) as the causative factor and marker of vascular injury related to insulin resistance. Postepy Hig Med Dosw (online). v. 70, n. 0, p. 1267-1275, Dez., 2016.

MANSOUR, M. et al. Metabolic syndrome and cardiovascular risk factors in obese adolescent. Open Access Macedonian Journal of Medical Sciences, Macedonia, v. 4, n. 1, p. 118-121, 2016. 
MARIE, N. G. et al. Global, regional, and national prevalence of overweight and obesity in children and adults during 1980-2013: a systematic analysis for the Global Burden of Disease Study 2013. The lancet, Londres, v. 384, n. 9945, p. 766-781, 2014.

MILLER, S. A., DYKES, D. D., POLESKY, H. F. A simple salting out procedure for extracting DNA from human nucleated cells. Nucleic acids Research, Oxford, v. 16, n. 3, p. 1215, 1988.

MORENO, M. et al. Circulating irisin levels are positively associated with metabolic risk factors in sedentary subjects. Plos one. v. 10, n. 4, p. 1-11, Abr., 2015.

MUDIE, K. et al. How does the association of general and central adiposity with glycaemia and blood pressure differ by gender and area of residence in a Malawian population: a cross-sectional study. International Journal of Epidemiology, v. 0, n. 0, 2018.

NOVELLE, M. G., CONTRERAS, C., ROMERO-PICÓ, A. Irisin, two years later. International Journal of Endocrinology, p. 1-8, 2013.

OLIOSA, P. R. et al. Body fat percentage is better than indicators of weight status to identify children and adolescents with unfavorable lipid profile. Jornal de Pediatria, v. 0, n. 0, 2017.

ORNELLAS, F. et al. Obese fathers lead to an altered metabolism and obesity in their children in adulthood: review of experimental and human studies. Jornal de Pediatria, v. 93, n. 6, p. 551-559, 2017.

PEREIRA, P. A. et al. Lack of association between genetic polymorphism of FTO, AKT1 and AKTIP in childhood overweight and obesity. Jornal de Pediatria. v. 92, n. 5, p. 521-7, Set/Out, 2016.

PIANTINO, C. B. et al. Parâmetros bioquímicos: associação com o estado nutricional e presença de patologia. Revista Brasileira de Obesidade, Nutrição e Emagrecimento, v. 11, n. 62, 2017.

ROTH, C. L. JAIN, V. Rising Obesity in Children: A Serious Public Health Concern. The Indian Journal of Pediatrics, v. 0, n. 0, 2018.

SANCHIS-GOMAR, F. GARATACHEA, N. PAREJA-GALEANO, H. et al. FNDC5 (irisin) gene and exceptional longevity: a functional replication study with rs16835198 and rs726344 SNPs. Official Journal of the American Aging Association (AGE), Grandville, v. 35, n. 9733, 2014.

SCOTT, W. R. et al. Investigation of Genetic Variation Underlying Central Obesity amongst South Asians. Plos One, v. 11, n. 5, Mai., 2016.

SLAUGTHER, M. H. et al. Skinfold equations for estimation of body fatness in children and youth. Human Biology, Washington, v. 60, n. 5, p. 709-723, 1988.

STIEFEL, E. C. et al. The prevalence of obesity and elevated blood pressure in adolescent student athletes from the state of Mississippi. Orthopaedic Journal of Sports Medicine, Thousand Oaks, v. 4, n. 2, p. 1-9, 2016.

TANG, S. et al. An Interaction between a FNDC5 Variant and Obesity Modulates Glucose Metabolism in a Chinese Han Population. Plos One, San Francisco, v. 9, n. 11, 2014.

WHO. World Health Organization. Growth reference data for 5-19 years. 2007. Disponível em: http://www.who.int/growthref/en/. Acesso em: 21 Jun. 2017.

YEH, J. et al. Obesity and Management of Weight Loss. The New England Journal of Medicine, Boston. v. 375, n. 12, p. 1187-9, Set., 2016.

YIN, R. Several genetic polymorphisms interact with overweight/obesity to influence serum lipid levels. Cardiovascular Diabetology, v. 11, n. 123, p. 1-18, 2012.

ZHAO, X. Y. et al. Risk of obesity-related gene polymorphism on the incidence and durative of childhood obesity. Zhonghua Liuxingbingxue Zazhi, v. 34, n. 6, p. 560-565, 2013.

ZHU, X. et al. Prevalence and demographic correlates of overweight, physical activity, and screen time among school-aged children in urban China: the Shanghai study. Asia Pacific Journal of Public Health, v. 0, n. $0,2018$. 